\title{
Higgs recoil analysis and Higgs width measurement at CEPC
}

\section{Zhenxing Chen ${ }^{* a}$ and Manqi Ruan ${ }^{b \dagger}$}

${ }^{a}$ State Key Laboratory of Nuclear Physics and Technology, Peking University, Beijing - China

${ }^{b}$ Institute of High Energy Physics, CAS, Beijing - China

E-mail: zxchen@ihep.ac.cn, manqi.ruan@ihep.ac.cn

The Circular Electron Positron Collider (CEPC) is a future Higgs factory proposed by the Chinese high energy physics community. It will operate at a center-of-mass energy of $240-250 \mathrm{GeV}$ and produce Higgs bosons via the $Z H$ process dominantly. Based on the recoil mass method, the expected accuracies of $Z H$ cross section $\sigma_{Z H}$, the Higgs mass $m_{H}$ and the Higgs width $\Gamma_{H}$ are studied at the CEPC with a MC sample corresponding to $5 a b^{-1}$ integrated luminosity. Without using any information from the Higgs decay, the absolute value of $\sigma_{Z H}$ could be measured to a relative precision of $0.50 \%$. In a model-dependent analysis, the $m_{H}$ precision is determined to be $5.0 \mathrm{MeV}$. The $\Gamma_{H}$ is determined by a series of individual analyses and its uncertainty is determined to be $2.8 \%$ at the CEPC.

38th International Conference on High Energy Physics

3-10 August 2016

Chicago, USA

*Speaker.

†n behalf of the CEPC study group. 


\section{Introduction}

After the discovery of Higgs boson at the LHC [1, 2], a series of further researches indicate that the Higgs boson is highly Standard Model (SM) like [3]. On the other hand, the percent level deviations are predicted in many new physics. Thus the percent or even sub-percent level precision becomes necessary for the future Higgs measurement. However, this uncertainty is difficult to achieve at the LHC [4]. Moreover, the Higgs boson can only be reconstructed by its decay products at the LHC and thus the Higgs total width or absolute couplings can not be determined in a modelindependent manner.

The CEPC is a Higgs factory proposed by the Chinese high energy physics community [5]. It will operate at a center-of-mass energy of $240-250 \mathrm{GeV}$ with an instantaneous luminosity of $2 \times 10^{34} \mathrm{~cm}^{-2} \mathrm{~s}^{-1}$ at each interaction point. The CEPC will accumulate about one million Higgs events, corresponding to an integrated luminosity of $5 \mathrm{ab}^{-1}$.

At the CEPC, the Higgs bosons are dominantly produced via the process of $e^{+} e^{-} \rightarrow Z H$ (Higgsstrahlung). By tagging the decay produces of associated $Z$ boson, the Higgsstrahlung events can be reconstructed with the recoil mass method:

$$
M_{\text {recoil }}=\sqrt{s+M_{f \bar{f}}^{2}-2\left(E_{f}+E_{\bar{f}}\right) \sqrt{s}}
$$

where $f \bar{f}$ represents the fermion pair from the $Z$ boson decay, $E_{f}$ and $E_{\bar{f}}$ are their energies, $M_{f \bar{f}}$ is the invariant mass, and $s$ is the square of center-of-mass energy. Thus the statistical precisions of $\sigma_{Z H}$ and $m_{H}$ can be determined by a fit to the $M_{\text {recoil }}$ distribution in a model-independent manner. The $m_{H}$ could be further improved by using the Higgs decay information.

The Higgs total width is a sensitive probe to new physics beyond the SM. For a SM Higgs boson of $125 \mathrm{GeV}$, its width is $4.1 \mathrm{MeV}$, which is far beyond the current detector resolution. However, the $\Gamma_{H}$ can be determined indirectly by the Higgs boson production cross sections and the corresponding branching ratios at an $e^{+} e^{-}$collider. In the first method, the $\Gamma_{H}$ is determined by

$$
\Gamma_{H}=\frac{\Gamma\left(H \rightarrow Z Z^{*}\right)}{B R\left(H \rightarrow Z Z^{*}\right)} \propto \frac{\sigma_{Z H}}{B R\left(H \rightarrow Z Z^{*}\right)}
$$

where $\Gamma\left(H \rightarrow Z Z^{*}\right)$ is the partial width of the Higgs boson decaying to $Z Z^{*}$ and $B R\left(H \rightarrow Z Z^{*}\right)$ is its branching ratio. In an alternative way, the $\Gamma_{H}$ can be determined by the cross section of the $W W$ fusion process $e^{+} e^{-} \rightarrow v \bar{v} H \rightarrow v \bar{v} b \bar{b}$, the branching ratios of $H \rightarrow b \bar{b}$ and $H \rightarrow W W^{*}$ :

$$
\Gamma_{H} \propto \frac{\Gamma(H \rightarrow b \bar{b})}{B R(H \rightarrow b \bar{b})} \propto \frac{\sigma_{v \bar{v} H \rightarrow v \bar{v} b \bar{b}}}{B R(H \rightarrow b \bar{b}) \cdot B R\left(H \rightarrow W W^{*}\right)}
$$

where $B R(H \rightarrow b \bar{b})$ and $B R\left(H \rightarrow W W^{*}\right)$ are the branching ratios of the Higgs boson decaying to $b \bar{b}$ and $W W^{*}$ respectively. These branching ratios are extracted from Higgsstrahlung process. Due to the small branching ratio of $B R\left(H \rightarrow Z Z^{*}\right)\left(2.3 \%\right.$ for a $125 \mathrm{GeV}$ SM Higgs boson), the $\Gamma_{H}$ precision in the first method is limited by the statistics of $H \rightarrow Z Z^{*}$ events. In the second method, the $\Gamma_{H}$ precision is limited by the discriminating power between $e^{+} e^{-} \rightarrow v \bar{v} H \rightarrow v \bar{v} b \bar{b}$ process and $e^{+} e^{-} \rightarrow Z H \rightarrow v \bar{v} b \bar{b}$. The final $\Gamma_{H}$ precision is determined by a combination of these two methods. 


\section{Conceptual Detector and Monte Carlo Simulation}

The CEPC conceptual detector geometry basically follows the design of International Linear Detector (ILD) at the International Linear Collider (ILC) [6, 7]. In order to accommodate the CEPC collision environment, some necessary changes have been made to the Machine Detector Interface and subdetector design. With respect to ILD, the CEPC conceptual detector has a $L^{*}$ (the distance between the interaction point and QD0, the final focusing magnet) of $1.5 \mathrm{~m}$, which is significantly shorter than that of the ILC $(4.5 \mathrm{~m})$. Besides, the CEPC has multiple interaction points and thus the push-pull operation is not necessary. So the thickness of return Yoke at the CEPC is reduced by 1 meter. More details about the conceptual detector can be found in reference [5].

The Monte Carlo samples, corresponding to an integrated luminosity of $5 a b^{-1}$, are generated with Whizard $1.95[8,9]$, with $\sqrt{s}=250 \mathrm{GeV}$ and $m_{H}=125 \mathrm{GeV}$. The SM backgrounds consist of 2-fermion processes ( $e^{+} e^{-} \rightarrow f \bar{f}$, where $f \bar{f}$ refers to all lepton and quark pairs) and 4-fermion processes, categorized as $Z Z, W W$, single $Z\left(e^{+} e^{-} Z\right)$ and single $W\left(e^{+} v_{e} W^{-}\right.$or $\left.e^{-} \bar{v}_{e} W^{+}\right)$. The radiation effects including ISR and FSR are considered in the generation. More details about the sample generation can be found in reference [10]. The Higgs signal samples are fully simulated with Mokka [11] and reconstructed with ArborPFA [12]. The background samples are processed with fast simulation, where the momentum resolution and detection efficiency are parameterized and validated for different types of particle.

\section{The Measurement of $Z H$ Cross Section and Higgs Mass}

The Higgs bosons are dominantly produced by the $e^{+} e^{-} \rightarrow Z H$ process at the CEPC. By tagging the decay products of associated $Z$ boson, the Higgs candidates are expected to present a peak in the recoil mass of $Z$ candidates. The $Z \rightarrow l^{+} l^{-}$decays can be easily identified and the lepton momenta can be precisely measured, so the events with leptonic $Z$ decays are ideal to reconstruct the $Z H$ recoil mass spectrum. A fit to the $M_{\text {recoil }}$ reveals the statistical uncertainty of $\sigma_{Z H}$ and $m_{H}$. In this case, there is no Higgs decay information used in the analysis, corresponding to a modelindependent measurement. The precision of Higgs boson can be further improved by taking into the Higgs decay products.

At current stage, the leptonic decay modes of $Z$ boson, including $Z \rightarrow e^{+} e^{-}$and $Z \rightarrow \mu^{+} \mu^{-}$, have been studied at the CEPC. These analyses are based on the full simulated $Z H$ signal and fast simulated backgrounds. In the $Z \rightarrow \mu^{+} \mu^{-}$channel, the leading background after event selection are $Z Z, W W$ and $Z \gamma$ (ISR return) events. In the $Z \rightarrow e^{+} e^{-}$channel, the additional backgrounds are from $e^{+} e^{-} \rightarrow e^{+} e^{-}(\gamma), e v W$ and eeZ. The $M_{\text {recoil }}$ distributions are shown in Fig. 1. The relative statistical precision of $\sigma_{Z H}$ in the $e^{+} e^{-}$channel and $\mu^{+} \mu^{-}$channel are $1.49 \%$ and $0.92 \%$ respectively. In order to improve the measurement precision of $m_{H}$, it is required there are more than two charged tracks in the final state. Then the $m_{H}$ precisions are $19.2 \mathrm{MeV}$ and $6.5 \mathrm{MeV}$ respectively. A combination of the two leptonic channels determines the $\sigma_{Z H}$ precision to be $0.50 \%$ and $m_{H}$ to be $5.0 \mathrm{MeV}$.

\section{The Measurement of Higgs Width}

For the process of $Z H\left(H \rightarrow Z Z^{*}\right)$, there are three bosons in the final state with one of them 

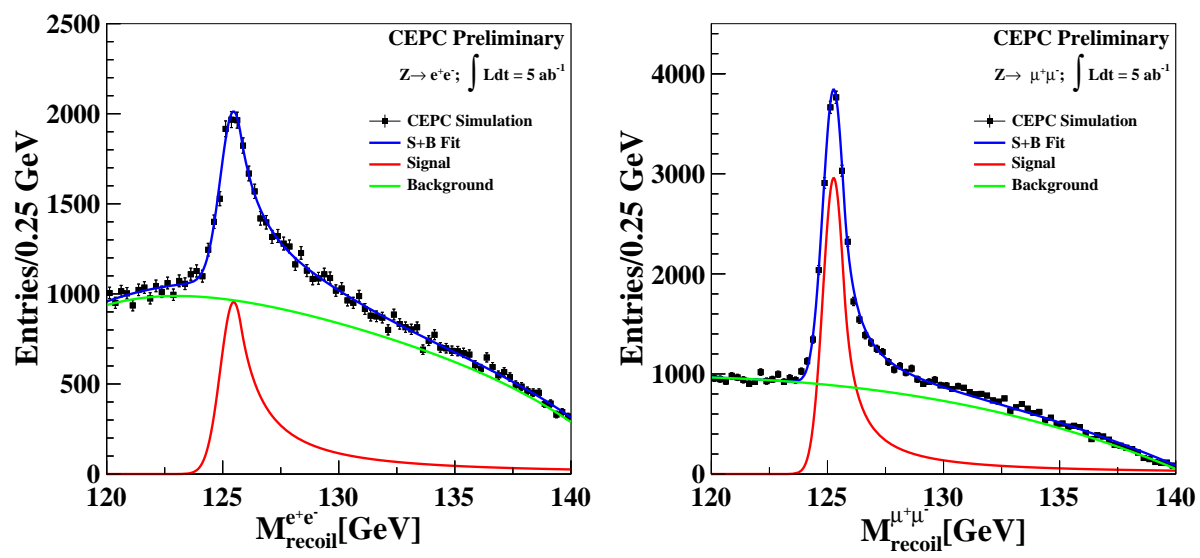

Figure 1: The recoil mass distribution for the $Z \rightarrow e^{+} e^{-}$and $Z \rightarrow \mu^{+} \mu^{-}$channels.

being off-shell. Consequently, this channel has a vary rich variety of topologies. At current stage, the tools of $\tau$ finder and jet clustering at the CEPC are under development. The final states with the initial $Z$ decaying to $e^{+} e^{-} / \mu^{+} \mu^{-}$and $Z Z^{*}$ decaying to $q \bar{q} \nu \bar{v}$ and $\mu^{+} \mu^{-} q \bar{q}$ have been studied with full simulation samples. The isolated leptons are reconstructed first and then the remaining reconstructed objects are produced from the jets. At an $e^{+} e^{-}$collider, the initial state is well known so the momenta of $v \bar{v}$ final state can be reconstructed by the momenta of initial state subtracted by the momenta of all reconstructed objects. The results for each channel are summarized in Table 1. The combined precision for these four channels is $5.4 \%$.

\begin{tabular}{|c|c|}
\hline$Z / Z Z^{*}$ & $\delta \sigma_{Z H} \times B R\left(H \rightarrow Z Z^{*}\right) / \sigma_{Z H} \times B R\left(H \rightarrow Z Z^{*}\right)(\%)$ \\
\hline$e^{+} e^{-} / q \bar{q} v \bar{v}$ & 12.7 \\
\hline$\mu^{+} \mu^{-} / q \bar{q} v \bar{v}$ & 7.0 \\
\hline$e^{+} e^{-} / \mu^{+} \mu^{-} q \bar{q}$ & 19.9 \\
\hline$\mu^{+} \mu^{-} / \mu^{+} \mu^{-} q \bar{q}$ & 15.5 \\
\hline Combined & 5.4 \\
\hline
\end{tabular}

Table 1: The result summary for $Z H\left(H \rightarrow Z Z^{*}\right)$.

For a SM Higgs boson with a mass of $125 \mathrm{GeV}$, there are nearly $70 \%$ of Higgs bosons decaying into a pair of jets: b-quarks (57.8\%), c-quarks (2.7\%) or gluons, gg (8.6\%). The analysis of $\mathrm{ZH}(H \rightarrow b \bar{b})$ is based on the measurement of $\mathrm{ZH}$ events with the Higgs boson decaying to two jets. Its precision is extracted by a template fit on the flavor tagging information of the two jets, including the b-jet likeliness and c-jet likeliness of each jet. According to the decay final states of the $Z$ boson in the $Z H$ events, the signal events are classified into di-lepton channel, neutrino channel and di-jet channel. The signal samples in each channel are full simulated. The backgrounds in the di-lepton channel are full simulated while the others are fast simulated. The precisions for each $Z$ boson decay mode are summarized in Table 2. The combined precision of $\sigma_{Z H} \times B R(H \rightarrow b \bar{b})$ is $0.2 \%$.

In the SM, the branching ratio of a Higgs boson at $125 \mathrm{GeV}$ decaying to $W W^{*}$ is about $21.5 \%$. The CEPC performance for the measurement of $\mathrm{ZH}\left(H \rightarrow W W^{*}\right)$ is examined with a study based 


\begin{tabular}{|c|c|}
\hline$Z \rightarrow$ & $\delta \sigma_{Z H} \times B R(H \rightarrow b \bar{b}) / \sigma_{Z H} \times B R(H \rightarrow b \bar{b})(\%)$ \\
\hline$e^{+} e^{-}$ & 1.3 \\
\hline$\mu^{+} \mu^{-}$ & 0.9 \\
\hline$v \bar{v}$ & 0.3 \\
\hline$q \bar{q}$ & 0.4 \\
\hline Combined & 0.2 \\
\hline
\end{tabular}

Table 2: The result summary for $Z H(H \rightarrow b \bar{b})$.

on fully simulated samples. It covers the channels of $Z$ boson decaying to $e^{+} e^{-}$and $\mu^{+} \mu^{-}$and $W W^{*}$ from the Higgs boson decaying to $l l v \bar{v}$ and $l v q \bar{q}$, where $l=e, \mu$. The event selection criteria contain the number of isolated leptons, jets, kinematic variables of the lepton system, missing energy and the impact parameter of lepton tracks (introduced to remove tau-related background). The combined precision of $\sigma_{Z H} \times B R\left(H \rightarrow W W^{*}\right)$ is $1.6 \%$. This result has been updated to $1.0 \%$ when the proceeding submitted. It is expected to be improved when other final states are considered.

In the measurement of $\sigma_{v \bar{v} H} \times B R(H \rightarrow b \bar{b})$, the main background includes $Z Z$ production and $Z H$ events with $Z \rightarrow v \bar{v}$ and $H \rightarrow b \bar{b}$. The latter is the dominant background and it interferes with the signal process. The interference effect is small and is ignored in this study. The $Z H$ events can be distinguished from $W W$ fusion by the recoil mass of $b \bar{b}$ system. However, the discriminating power is limited at a center-of-mass energy of $250 \mathrm{GeV}$. The analysis is based on fast simulation and the precision of $\sigma_{v \bar{v} H} \times B R(H \rightarrow b \bar{b})$ is determined to be $2.8 \%$.

Table 3 summarizes the expected measurement precisions related to Higgs width. The precisions obtained with Eq. 1.2 and Eq. 1.3 are 5.4\% and 3.3\% respectively. A combined result yields a relative precision of $2.8 \%$ with $5 a b^{-1}$ integrated luminosity.

\begin{tabular}{|c|c|}
\hline & Precision(\%) \\
\hline$\sigma_{Z H}$ & 0.5 \\
\hline$\sigma_{Z H} \times B R\left(H \rightarrow Z Z^{*}\right)$ & 5.4 \\
\hline$\sigma_{Z H} \times B R(H \rightarrow b \bar{b})$ & 0.21 \\
\hline$\sigma_{Z H} \times B R\left(H \rightarrow W W^{*}\right)$ & 1.6 \\
\hline$\sigma_{v \bar{v} H} \times B R(H \rightarrow b \bar{b})$ & 2.8 \\
\hline$\Gamma_{H}$ & 2.8 \\
\hline
\end{tabular}

Table 3: The result summary for Higgs width measurement.

\section{Summary}

In this report, we present the benchmark analyses of the measurements of $Z H$ cross section, the Higgs mass and the Higgs width at the CEPC. The Monte Carlo sample is set at a centerof-mass energy of $250 \mathrm{GeV}$ with an integrated luminosity of $5 a b^{-1}$. All the Higgs signal and the backgrounds in part of the measurements are fully simulated and reconstructed. In a modelindependent manner, the statistical precision of $Z H$ cross section $\sigma_{Z H}$ is determined to be $0.50 \%$ with the recoil mass method. With information from the Higgs decay, the precision of Higgs mass 
can be further improved to $5.0 \mathrm{MeV}$. The Higgs width $\Gamma_{H}$ is determined by a series of individual analyses, leading to a relative precision of $2.8 \%$. This result is expected to be improved when more final states are considered.

\section{References}

[1] G. Aad et al (The ATLAS Collaboration), Observation of a new particle in the search for the Standard Model Higgs boson with the ATLAS detector at the LHC, Phys. Lett. B 7161 (2012) [hep-ex/1207.7214]

[2] S. Chatrchyan et al (The CMS Collaboration), Observation of a new boson at a mass of $125 \mathrm{GeV}$ with the CMS experiment at the LHC, Phys. Lett. B 71630 (2012) [hep-ex/1207. 7235].

[3] G. Aad et al (The ATLAS and CMS Collaboration), Combined Measurement of the Higgs Boson Mass in pp pp Collisions at $\sqrt{s}=7$ and 8 TeV with the ATLAS and CMS Experiments, Phys. Rev. Lett. 114191803 (2015) [hep-ex/1503.07589].

[4] S. Dawson, A. Gritsan, H. Logan et al, Working Group Report: Higgs Boson, [hep-ex/1310.8361].

[5] M. Ahmad et al (The CEPC-SPPC Study Group), CEPC-SppC Preliminary Conceptual Design Report: Physics and Dector, http://cepc.hep.ac.cn/preCDR/main_preCDR.pdf

[6] T. Abe et al (The ILD concept group), The International Large Detector: Letter of Intent, [hep-ex/1006.3396].

[7] T. Behnke, J. Brau, P. Burrows et al, The International Linear Collider Technical Design Report Volume 4: Detectors, [hep-ex/1306.6329]

[8] W. Kilian, T. Ohl and J. Reuter, WHIZARD: Simulating Multi-Particle Processes at LHC and ILC, Eur. Phys. J. C 711742 (2011) [hep-ph/0708.4233].

[9] M. Moretti, T. Ohl and J. Reuter, O'Mega: An Optimizing matrix element generator, [hep-ph/0102195].

[10] Xin Mo, Gang Li, Manqi Ruan et al, Physics cross sections and event generation of $e^{+} e^{-}$ annihilations at the CEPC, Chin. Phys. C 40033001 (2016) [hep-ex/1505. 01008].

[11] P. Mora de Freitas and H. Videau, Detector simulation with MOKKA/GEANT4: Present and future, The International Workshop on Linear Colliders (LCWS 2002).

[12] Manqi Ruan, Arbor, a new approach of the Particle Flow Algorithm, [physics.ins-det/1403.4784]. 